\title{
Resonances and Fields near Metamaterial Inclusions
}

\author{
R.C. McPhedran \\ CUDOS, School of Physics \\ University of Sydney \\ Sydney, NSW, Australia \\ ross@physics.usyd.edu.au
}

\author{
J. Helsing \\ Centre for Mathematical Sciences \\ Lund University \\ Lund, Sweden \\ Johan.Helsing@na.lu.se
}

\author{
G.W. Milton \\ Department of Mathematics \\ University of Utah \\ Salt Lake City, Utah, USA \\ milton@math.utah.edu
}

\begin{abstract}
We consider systems of particles in two dimensions with negative dielectric permittivity and sharp corners. The field divergences near the corners of the inclusions are non-squareintegrable for negative permittivities in a range depending on the corner angle, and the leading term for small distances is provided by the static term. We have developed a highly accurate numerical method which uses adaptive techniques to resolve field behaviour near the corners. We use this to elucidate the resonant spectrum associated with the effective permittivity of arrays of such particles, and in particular its behaviour as the particles approach touching.
\end{abstract}

\section{INTRODUCTION}

The behaviour of electromagnetic fields near edges of dielectric bodies was discussed in an influential paper by $\mathrm{J}$. Meixner [1]. He showed the nature of the fields near edges, and gave a transcendental equation giving the power law governing field divergence in the neighbourhood of the edge. He showed that the lead term giving the field components near edges obeyed Laplace's equation rather than the Helmholtz equation (the rapid variation in the edge region rendering the term proportional to the wave number squared in the Helmholtz equation negligible).

Hetherington and Thorpe [2] considered the electrostatic fields in arrays of polygonal particles, and discussed the spectrum of their effective permittivity or polarizability. They showed that the resonant spectrum consisted of two parts: a continuous spectrum given by the same transcendental equation as Meixner, and a discrete spectrum lying in the remainder of the negative real permittivity line.

With the advent of the field of metamaterials, a number of authors have noticed that calculation of the electromagnetic properties of particles or systems of particles with sharp corners can lead to strong numerical instabilities when the permittivity or permeability of the particles is negative. At the same time, it has been realised that in certain applications of such systems, the use of particles with sharp corners and negative permittivities can be advantageous, for example in offering strong optical absorption and field concentrations over a wide bandwidth [3], [4].

Here, we discuss the application of a new method of adaptive solution of integral equations to the understanding of the nature of the fields near metamaterial inclusions, and of the resonant spectrum of systems of such particles. We will concentrate on the case of systems of square particles ap- proaching the chequerboard array, a difficult geometry which has been of interest to a number of authors.

\section{Numerical Method and Results}

We consider the calculation of electrostatic fields near the sharp corner of a dielectric object in two dimensions. Let us suppose that the electrostatic potential varies with distance $r$ from a corner with an included angle $2 \psi$ as $r^{\beta}$, and that the permittivity ratio inside the inclusion to that outside is $\sigma$. Then [1], [2] $\beta$ is found by solving a transcendental equation:

$$
\frac{\tan [\beta(\pi-\psi)]}{\tan (\beta \psi)}=-\sigma,
$$

or the same equation with $\sigma$ replaced by $1 / \sigma$. For $\sigma$ real, the solution $\beta$ of (1) is either pure real or pure imaginary.

To clarify the behaviour of fields, consider the case of $\psi=$ $\pi / 4$, corresponding to a $90^{\circ}$ corner. Then (1) gives

$$
\cos (\beta \pi / 2)=\frac{(\sigma-1)}{2(\sigma+1)} .
$$

From this, we see that the right-hand side exceeds one in magnitude for $\sigma$ lying between -3 and $-1 / 3$, and that $\beta$ is then pure imaginary, corresponding to a solution for the potential which oscillates with $r$, and the oscillations become ever more rapid as $r$ tends to zero. The electric field is given by the spatial derivative of the potential, and it diverges like $1 / r$ multiplied by the oscillating term as $r \rightarrow 0$. The branch cut region here is that where $\beta$ is imaginary, i.e., between $\sigma=-3$ and $\sigma=-1 / 3$. Hetherington and Thorpe [2] postulate that for an $m$ sided regular polygon, there will be a branch cut running between $\sigma=(2+m) /(2-m)$ and $\sigma=(2-m) /(2+m)$. They made this assertion after recognizing that in this interval of $\sigma$ the surface charge (rather that just the surface charge density) near the corner is infinite, which is unphysical.

To calculate fields accurately for this difficult problem, we apply an integral equation approach and use a novel numerical method called recursive compressed inverse preconditioning [5] . Conceptually this is a local multilevel technique which makes a change of basis and expresses the non-smooth solution to the single-layer integral equation in terms of a piecewise smooth transformed layer density which can be cheaply resolved by polynomials.

To illustrate the accuracy and stability of the method, we have constructed animations showing the evolution of the 
effective permittivity of the square and staggered square arrays of square cylinders, as the area fraction of the inclusions varies from zero until the inclusions almost touch (see [6]). Figure 1 shows a frame of one of our animations. It will be noticed that the inclusions have come very close to touching, but the numerical results are still stable and accurate. They show that there is a branch-cut of the effective permittivity function in the interval $[-3,-1 / 3]$ of the permittivity ratio; in this interval, the effective permittivity can have a one-sided complex limit as the imaginary part of the permittivity ratio tends down to zero. Away from the branch-cut, the effective permittivity function has a sequence of poles which become more and more dense as the squares come close to touching. Exactly at the touching area fraction, these poles become dense, with the result that the branch-cut fills the entire negative real axis of the permittivity ratio, as required by analytic results for the chequerboard.

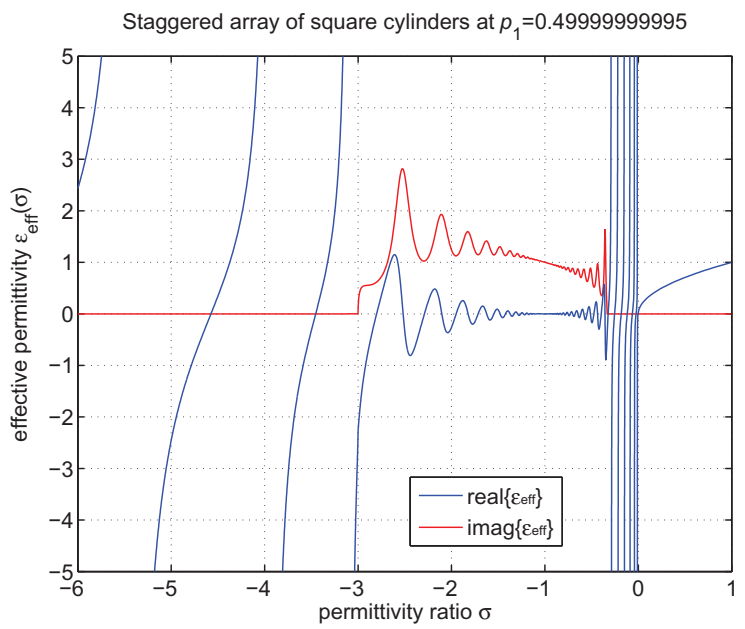

Fig. 1. The effective permittivity of a staggered square array very close to touching, as a function of permittivity ratio.

Poles and zeros are created from oscillations in the effective permittivity function $\epsilon_{\mathrm{eff}}$ as they leave the branch cut region. The oscillations themselves are created at the permittivity ratio $\sigma=-1$, which is an essential singularity of the function $\epsilon_{\text {eff }}(\sigma)$ for inclusions with smooth boundaries (although not for the inclusions with sharp corners considered here), and move outwards as the square cylinders approach each other. In this way the continuous spectrum "spawns" the discrete spectrum.

We will also present results giving further details on the way the field structures from the continuous part of the spectrum change as they move into the discrete part of the spectrum.

\section{REFERENCES}

[1] J. Meixner, "The behaviour of electromagnetic fields near edges", IEEE Trans, vol. AP20, pp. 442-446, July 1972.

[2] J.H. Hetherington and M.F. Thorpe, "The conductivity of a sheet containing sharp corners", Proc Roy Soc Lond, vol. A438, pp. 591-604, September 1992.

[3] R.C. McPhedran and W.T. Perrins, "Electrostatic and optical resonances of cylinder pairs", Appl. Phys., vol. 24, pp. 311-318, April 1981.
[4] A. Aubry, D.Y. Lei, A.I. Fernandez-Dominguez, Y. Sonnefraud, S.A. Maier and J.B. Pendry, "Plasmonic Light-Harvesting Devices over the Whole Visible Spectrum", Nano Letters , vol. 10, 2574-2579, October 2010.

[5] J. Helsing and R. Ojala, "Corner singularities for elliptic problems: Integral equations, graded meshes, quadrature, and compressed inverse preconditioning", J. Comput. Phys., vol. 227, 8820-8840, October 2008.

[6] J. Helsing, R.C. McPhedran and G.W. Milton, "Spectral super-resolution in metamaterial composites ", New Journal of Physics, vol. 13, $1367-$ 2630/11/115005+17, November 2011.

\section{ACKNOWLEDGEMENT}

Ross McPhedran acknowledges support from the Australian Research Council's Discovery Projects and Centre of Excellence Schemes. Johan Helsing acknowledges support from the Swedish Research Council through grant 621-2011-5516. Graeme Milton acknowledges support from the National Science Foundation through grant DMS-0707978. 